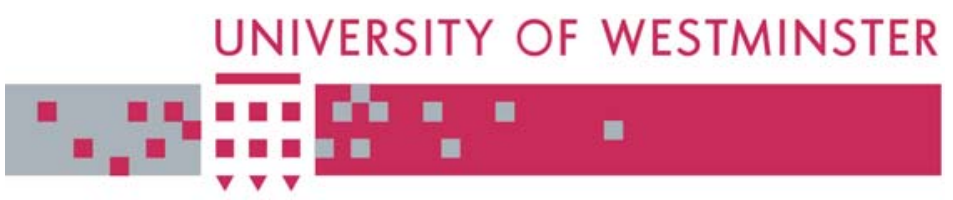

\title{
WestminsterResearch
}

http://www.wmin.ac.uk/westminsterresearch

\section{Patients flow: a mixed-effects modelling approach to predicting discharge probabilities.}

\author{
Shola Adeyemi \\ Thierry Chaussalet \\ Haifeng Xie \\ Peter Millard \\ School of Informatics
}

Copyright (C [2007] IEEE. Reprinted from the proceedings of the 20th IEEE International Symposium on Computer-Based Medical Systems. IEEE CBMS 2007, Maribor, Slovenia, 20-22 June 2007. IEEE, Los Alamitos, USA, pp. 725730. ISBN 0769529054.

This material is posted here with permission of the IEEE. Such permission of the IEEE does not in any way imply IEEE endorsement of any of the University of Westminster's products or services. Personal use of this material is permitted. However, permission to reprint/republish this material for advertising or promotional purposes or for creating new collective works for resale or redistribution to servers or lists, or to reuse any copyrighted component of this work in other works must be obtained from the IEEE. By choosing to view this document, you agree to all provisions of the copyright laws protecting it.

The WestminsterResearch online digital archive at the University of Westminster aims to make the research output of the University available to a wider audience. Copyright and Moral Rights remain with the authors and/or copyright owners.

Users are permitted to download and/or print one copy for non-commercial private study or research. Further distribution and any use of material from within this archive for profit-making enterprises or for commercial gain is strictly forbidden.

Whilst further distribution of specific materials from within this archive is forbidden, you may freely distribute the URL of the University of Westminster Eprints (http://www.wmin.ac.uk/westminsterresearch).

In case of abuse or copyright appearing without permission e-mail wattsn@wmin.ac.uk. 


\title{
Patients Flow: A Mixed-Effects Modelling Approach to Predicting Discharge Probabilities
}

\author{
Shola Adeyemi, Thierry Chaussalet, Haifeng Xie and Peter Millard \\ Health and Social Care Modelling Group, School of Informatics \\ University of Westminster, London, UK \\ s.adeyemi3@westminster.ac.uk, (chausst,xieh)@wmin.ac.uk,phmillard@tiscali.co.uk
}

\begin{abstract}
A mixed effects approach is hereby introduced to patients flow and length of stay modelling. In, particular, a class of generalized linear mixed models has been used to demonstrate the usefulness of this approach. This modelling technique is used to capture individual patients experience during the process of care as represented by their pathways through the system. The approach could predict the probability of discharge from the system, as well as detect where the system may be going wrong.
\end{abstract}

\section{Introduction}

Average length of stay (LOS) has been shown to be a good proxy for hospital resource consumption and disease severity [1]. In the literature, LOS in health and social care systems has been described using patient flow models. Modelling patients flow in health care systems is considered to be vital in understanding the operational and clinical functions of the system and has proved to be useful in improving the functionality of the health care system. These papers have used different techniques to model patient flow in health care systems with several different assumptions imposed on the system. Markov models have been used extensively to capture probabilistic laws that govern the dynamics of patients between states in the healthcare system. A two-stage continuous-time Markov model that describes the movement of patients through two compartments in geriatric hospitals was developed in [2]. Such an approach takes into account, conceptually, different types of patients and their corresponding length of stay. The (Markov) model [2] was extended to describe the behaviour of patients moving through three stages in a geriatric department [3]. A Markov model in continuous time for the LOS of elderly people moving within and between residential home care and nursing home care has also been developed using the framework of aggregated Markov processes [4]. Phase-type distributions have also been employed to represent the variable nature of LOS. This class of distributions describes the time to absorption of a finite Markov chain in continuous time, where there is a single exit (absorbing state) and the stochastic process starts in a transient state, [5]. Figure 1, below, describes the Coxian phase-type distribution where the absorbing state is discharge by death.

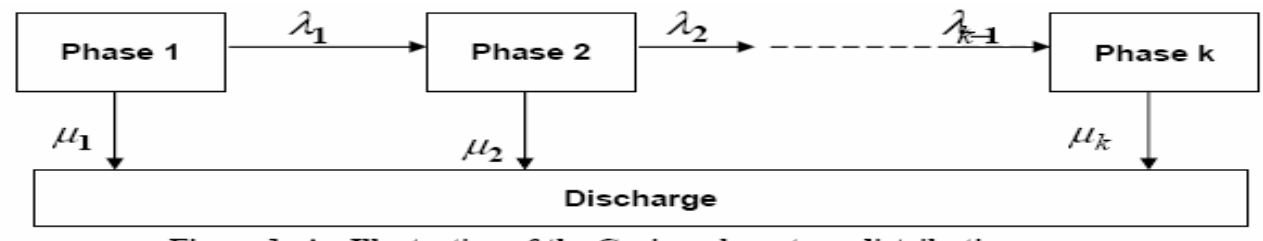

Figure 1: An Illustration of the Coxian phase-type distribution 
These models describe duration until an event occurs in terms of a process consisting of a sequence of latent phases- the states of a latent Markov model. However, the generality of phase-type distributions makes it difficult to estimate all the parameters of the model. To overcome this problem Coxian Phase-type distributions were introduced. Coxian phasetype distributions describe duration until an event in terms of a process which consists of a sequence of latent phases. Compartmental and simulation modelling has been an approach for modelling LOS [6], The compartmental framework has also been incorporated into queuing theoretical approach however, due to the complexity, discrete event simulation (DES) has been employed to solve the system equations [7]. It should be stressed that the compartments were virtual therefore the analyses could only be considered a hypothetical system rather than a real one.

In almost all studies of patient flow and LOS modelling what has been overlooked is what an individual patient experiences during the delivery of care [8]. In order to have an insight into what an individual patient experience during the course of care, we seek to develop a mixed modelling framework for patient flow paths through the healthcare system. This approach could predict the probability of discharge from the system, as well as detect where there may be problems with flow.

\section{Modelling Approach}

In the healthcare system, either the improvement of patients depends on activities (or interventions) in each state visited in the care process or the recovery of patients progresses in stages (states) until discharge. According to [9], this represents patient journeys (flow paths) that a particular clinical process may entail which make patients pass through the health system and attain different health-states. These can, for example, also reflect the physical progression of patients between different hospital locations. Since our aim is to model the experience of individual patients in the healthcare system, these experiences are the different states (locations or health states) visited captured by individual patient's flow paths through the system. In a multi-state system, the flow paths represent clustered or repeated observations within an individual patient. When repeated measurements are taken on patients, classical regression assumptions are violated; therefore mixed models are developed to model the outcome in a view to capture the correlation structure induced and the patient specific random effects. There are two distinct approaches to the analysis. First, the heterogeneity can be explicitly modelled; we will refer to this as the 'patient-specific' approach. These patient specific effects are assumed to follow a parametric distribution across population, usually normal. Second, the populationaveraged response can be modelled as a function of covariates without explicitly accounting for patient to patient heterogeneity. A mixed effect model satisfies, [10]

$\mathbf{Y}=\mathbf{X} \boldsymbol{\beta}+\mathbf{Z b}+\mathbf{\varepsilon}$

where

$\mathbf{Y}$ is the vector of different combinations of path ways

$\mathbf{X}$ is the design matrix for the fixed (patients' population) effects

$\boldsymbol{\beta}$ is the vector of fixed (average patients' population) effect parameters

$\mathbf{Z}$ is the design matrix for the random (patient specific) effects

$\mathbf{b}$ is the vector of random (patient specific) effects

$\boldsymbol{\varepsilon}$ is the vector of experimental (measurement) errors 
The regression coefficients (fixed effect parameters) have interpretations for the population rather than for any individual. If $\mathrm{Y}$ is Gaussian, the model is referred to as a linear mixed model while a generalized linear mixed model whenever $Y$ is non-Gaussian. The linear mixed model and the generalized linear mixed models are linear both in the predictor and the parameter however when $\mathrm{Y}$ is non-Gaussian and the mean is modelled as a nonlinear function of the parameters and the predictor, the model (2.1) is referred to as a nonlinear mixed model. In this paper, we demonstrate a class of the generalized linear mixed models.

\section{Illustration of the Approach}

The figure below depicts activities within an artificial system. This system assumes that patients entering into the system will undergo some interventions and then progress from doing primary, secondary and discharge planning activities before discharge. Discharge can be by death, discharge to patients' home or transfer to another healthcare institution. For simplicity we have forced each state of the system to have three exits, this is not a restriction for using this approach however, at least two exit paths are necessary. The coding of the paths are ordered categories which gives the highest level of improvement by patients for state $i$ as ${ }_{i 2}=2$, and ${ }_{i 1}=1$ while ${ }_{i 0}=0$ is the lowest level of improvement.

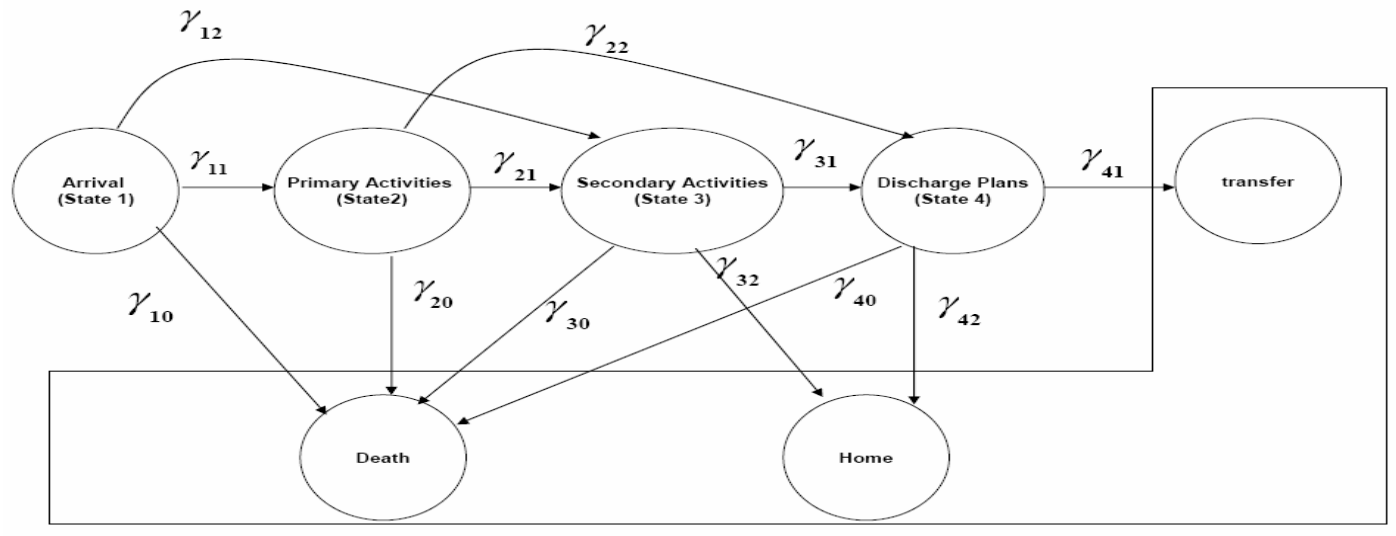

Figure 2: Disease progression through an artificial system- Adapted from Cote 2000

The probability distribution of potential exit (or discharge) from the states through any of the paths ( ${ }_{i j}, i=1, \ldots, 4$ and $\left.j=0,1,2\right)$ in Figure 1 , is modelled by

$$
p y i=\frac{\exp \sum_{u=0}^{y}\left(\begin{array}{ll}
p & \\
\text { iu }
\end{array}\right)}{\sum_{k=0}^{m_{i} 1} \exp \sum_{u=0}^{k}\left(\begin{array}{ll}
p & \\
m_{i u}
\end{array}\right)} ; \quad y=0,1, \ldots \ldots \ldots, m_{i} \quad 1 .
$$

The probability of patient $p$ passing through $y$ (i.e. through any ${ }_{i j}$ ) on the $m_{i}$-path state $i$ is a function of the person's position on $\theta$ (person specific random effect) and the difficulties of the $m_{i}$ paths in state $i$. The observation $y$ can be seen as a count of surpassed paths, and only 
the difficulties of these $y$ surpassed paths appear in the numerator of the model. The score, number of surpassed paths in the system, for each patient is a sufficient statistics for $\boldsymbol{\theta}_{p}$, [11] distributed normally with mean 0 and variance ${ }^{2}$. The ${ }_{i}$ s show the relative ease (or difficulty) of passing through each state by different patients having person specific random effect (e.g. health status). These random effects scaled between -3 and +3 .

The Table below gives the parameter estimates for the model proposed in (1) based on maximum likelihood estimation using non-adaptive Gaussian quadrature with 20 nodes and implemented in the SAS V9.

Table 1: Parameter estimates for the model presented in (1)

\begin{tabular}{|c|l|l|l|l|l|}
\hline Parameter & Estimate & Standard Error & Degree of Freedon & T Value & p-values \\
\hline 1 & -1.7047 & 0.4671 & 59 & -3.65 & 0.0006 \\
\hline 2 & 0.6931 & 0.2582 & 59 & 2.68 & 0.0094 \\
\hline 3 & 2.3026 & 0.3464 & 59 & 6.65 & $<0.0001$ \\
\hline 4 & 1.7918 & 0.2981 & 59 & 6.01 & $<0.0001$ \\
\hline
\end{tabular}

Presented in the figures (Figures $3-6$ ) below are the discharge curves for each state. These curves are model based i.e. obtained from the estimated parameters. On the x-axis are the patient specific random effects theta and some threshold parameters. These figures give the probability of discharge with respect to the location of patients on the health status (theta) scale. Because each person can only pass through one path and the three options are mutually exclusive, the sum of the three probabilities at a particular value of theta is 1 . The probability of discharge, from the system, with respect to the location of patients on the health status (theta) scale can be calculated. Suppose ${ }_{p i}$ is the value of the random effect for patient $p$ in state $i$ and ${ }_{i j}$ is the observable (ordinal variate) paths $j$ through $i$. Under the model, there are thresholds values, ${ }_{i 1}$, and ${ }_{i 2}$ such that

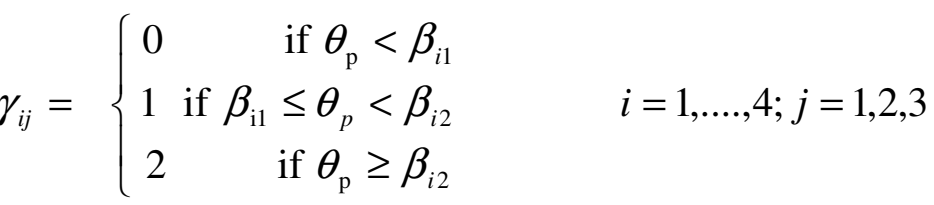

For instance, observing state 1 (Figure 3), path 0 and path 1 intersect at ${ }_{i 1}=1.5$ and path 1 and path 2 intersect at ${ }_{i 2}=0.1$.

These thresholds show that the most likely path is path 0 below ${ }_{i 1}$ and path1 is the most likely between ${ }_{i 1}$ and ${ }_{i 2}$ while path2 is the most likely above ${ }_{i 2}$. Note that if ${ }_{i 2}<{ }_{i 1}$, the interpretation changes with path 1 not being most likely at any point. This might be interpreted as indicating a problem with path 1, but this is not always true. State2 (Figure 4), shows the threshold have changed. ${ }_{i 1}$ has changed from -1.5 in state 1 (Figure 3) to -0.6 
and $\quad{ }_{i 2}$ remains fixed at 0.1 . These thresholds serve as indicators of possible paths that can be taken by a patient given the person specific random effect and give a way of predicting the success of interventions in the states for any new patient.

Observing state 3 (Figure 5), $i_{i 2}<{ }_{i 1}$ which may be interpreted that path 1 may not be needed in the system since patients who are not dead and have patient specific random effect above ${ }_{i 1}$ could be discharged from state 3 to recuperate in their own home. This is an indication that this modelling technique can detect where the system may be going wrong.
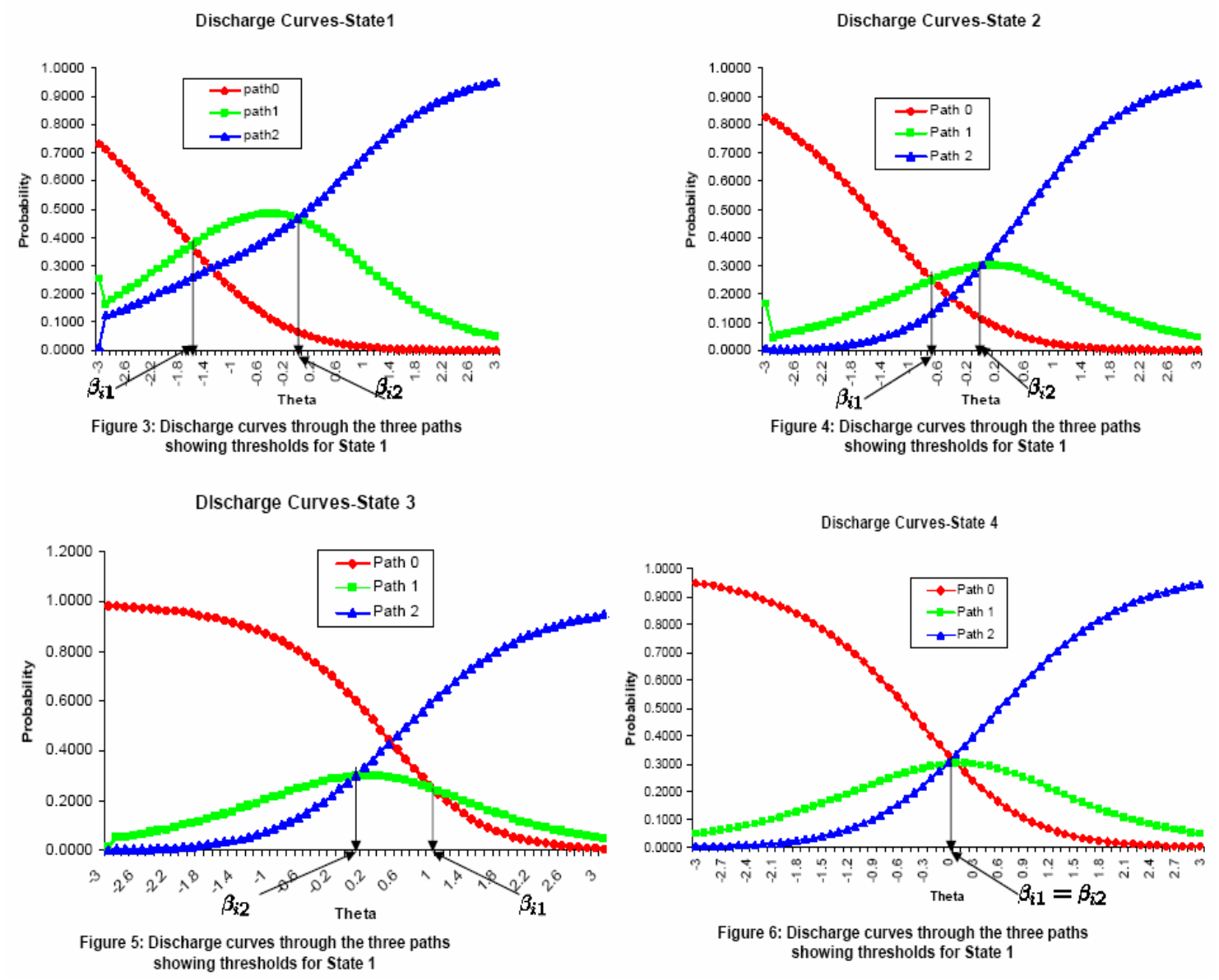

For state 4 (Figure 6) ${ }_{i 1}=i_{i 2}=0$, this is a point at which it is equally likely for any patient to pass through any of the paths. Points less than $i_{i 1}=i_{i 2}=0$ are more likely to pass through path 0 and path 2 is more likely above the threshold. Another interpretation is that patients below the population average are more likely to pass through path 0 while patients whose health status is greater than the population average are more likely to pass though the path 2 . Discharge probabilities from the system through any combination of paths can easily be calculated. 


\section{Conclusion}

Previous work have independently model LOS without capturing the flow paths of patients through the system. However, in this paper we have demonstrated the fact that mixed models are useful in health and social care modelling since patient experience in the care process is highly important for improvement of the process of care and better planning. We have presented a model for the prediction of discharge probabilities based on individual patient's flow paths (trajectories) and patient-specific random effects. However, an elegant way will be to jointly model LOS and patients flow paths. Therefore, an extension of this work to the joint modelling of LOS and individual patient's flow paths is currently being investigated.

\section{References}

[1] E. Kulinskaya, D. Kornbrot, and H. Gao, "Length of stay as a performance indicator: robust statistical methodology," IMA J Management Math, vol. 16, pp. 369-381, 2005.

[2] V. Irvine, S. Mcclean, and P. Millard, "Stochastic-Models for Geriatric Inpatient Behavior," IMA Journal of Mathematics Applied in Medicine and Biology, vol. 11, pp. 207-216, 1994.

[3] S. Mcclean and P. Millard, "Patterns of Length of Stay after Admission in Geriatric-Medicine - an Event History Approach," Statistician, vol. 42, pp. 263-274, 1993.

[4] H. Xie, T. J. Chaussalet, and R. H. Millard, "A continuous time Markov model for the length of stay of elderly people in institutional long-term care," Journal of the Royal Statistical Society Series a-Statistics in Society, vol. 168, pp. 51-61, 2005.

[5] M. J. Faddy and S. I. McClean, "Analysing data on lengths of stay of hospital patients using phase-type distributions," Applied Stochastic Models in Business and Industry, vol. 15, pp. 311-317, 1999.

[6] G. W. Harrison and P. H. Millard, "Balancing Acute and Long-Term Care - the Mathematics of Throughput in Departments of Geriatric-Medicine," Methods of Information in Medicine, vol. 30, pp. 221-228, 1991.

[7] E. El-Darzi, C. Vasilakis, T. Chaussalet, and P. H. Millard, "A simulation modelling approach to evaluating length of stay, occupancy, emptiness and bed blocking in a hospital geriatric department," Health Care Management Science, vol. 1, pp. 143-149, 1998.

[8] M. J. Cote and W. E. Stein, "A stochastic model for a visit to the doctor's office," Mathematical and Computer Modelling, vol. 45, pp. 309-323, 2007.

[9] M. J. Cote and W. E. Stein, "An Erlang-based stochastic model for patient flow," OmegaInternational Journal of Management Science, vol. 28, pp. 347-359, 2000.

[10] N. M. Laird and J. H. Ware, "Random-Effects Models for Longitudinal Data," Biometrics, vol. 38, pp. 963-974, 1982.

[11] G. N. Masters, "A Rasch Model for Partial Credit Scoring," Psychometrika, vol. 47, pp. 149$174,1982$. 